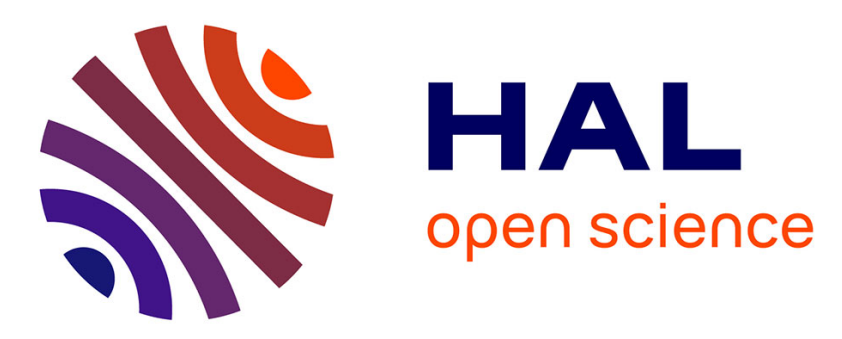

\title{
The influence of capacity- and time-constrained intermediate storage in two-stage food production systems
}

Renzo Akkerman, Dirk Pieter van Donk, Gerard J C Gaalman

\section{- To cite this version:}

Renzo Akkerman, Dirk Pieter van Donk, Gerard J C Gaalman. The influence of capacity- and time-constrained intermediate storage in two-stage food production systems. International Journal of Production Research, 2007, 45 (13), pp.2955-2973. 10.1080/00207540600806463 . hal-00512926

\section{HAL Id: hal-00512926 \\ https://hal.science/hal-00512926}

Submitted on 1 Sep 2010

HAL is a multi-disciplinary open access archive for the deposit and dissemination of scientific research documents, whether they are published or not. The documents may come from teaching and research institutions in France or abroad, or from public or private research centers.
L'archive ouverte pluridisciplinaire HAL, est destinée au dépôt et à la diffusion de documents scientifiques de niveau recherche, publiés ou non, émanant des établissements d'enseignement et de recherche français ou étrangers, des laboratoires publics ou privés. 


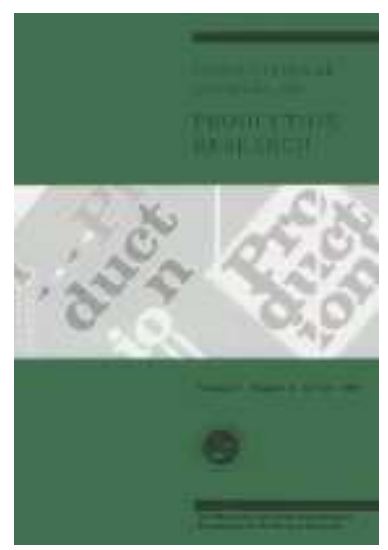

The influence of capacity- and time-constrained intermediate storage in two-stage food production systems

\begin{tabular}{|c|c|}
\hline Journal: & International Journal of Production Research \\
\hline Manuscript ID: & TPRS-2006-IJPR-0100 \\
\hline Manuscript Type: & Original Manuscript \\
\hline $\begin{array}{r}\text { Date Submitted by the } \\
\text { Author: }\end{array}$ & 09-Feb-2006 \\
\hline Complete List of Authors: & $\begin{array}{l}\text { Akkerman, Renzo; University of Groningen, Faculty of Management } \\
\text { and Organization } \\
\text { Van Donk, Dirk Pieter; University of Groningen, Faculty of } \\
\text { Management and Organization } \\
\text { Gaalman, Gerard J C; University of Groningen, Faculty of } \\
\text { Management and Organization }\end{array}$ \\
\hline Keywords: & $\begin{array}{l}\text { OPERATIONS MANAGEMENT, PROCESS INDUSTRY, SIMULATION, } \\
\text { PRODUCTION MANAGEMENT }\end{array}$ \\
\hline Keywords (user): & intermediate storage \\
\hline
\end{tabular}

\section{S) ScholarONE \\ Manuscript Central}




\title{
The influence of capacity- and time-constrained intermediate storage in two-stage food production systems
}

\author{
Renzo AKKerman, Dirk Pieter VAN DONK, Gerard GAALMAN \\ Production Systems Design Group \\ Faculty of Management and Organization \\ University of Groningen \\ The Netherlands
}

\author{
Corresponding author: \\ R. Akkerman \\ University of Groningen \\ Faculty of Management and Organization \\ P.O. Box 800 \\ 9700 AV Groningen \\ The Netherlands \\ Tel: +31503637924 \\ Fax: +31 503632032 \\ r.akkerman@rug.nl
}




\title{
The influence of capacity- and time-constrained intermediate storage in two-stage food production systems
}

\begin{abstract}
In food processing, two-stage production systems with a batch processor in the first stage and packaging lines in the second stage are common and mostly separated by capacity- and time-constrained intermediate storage. This combination of constraints is common in practice, but literature hardly pays attention to this.

In this paper, we show how various capacity and time constraints influence the performance of a specific two-stage system. We study the effects of several basic scheduling and sequencing rules in the presence of these constraints in order to learn the characteristics of systems like this.

Contrary to the common sense in operations management, the LPT rule is able to maximize the total production volume per day. Furthermore, we show that adding one tank has considerable effects. Finally, we conclude that the optimal setup frequency for batches in the first stage is dictated by the storage time constraint.
\end{abstract}

Keywords: food processing; storage tanks; capacity constraints; time constraints; simulation 


\section{Introduction}

In the food-processing industry, production systems often consist of two stages. In general, the first stage concerns the batch processing of raw material into food products, which are packaged in the second stage.

In previous research (e.g. Van Donk, 2001), perishable goods, shared resources (such as tanks), and a divergent product structure were identified (among others) as important characteristics of food processing. These characteristics imply the presence of two types of intermediate storage limitations: capacity constraints and time constraints.

The capacity constraints are present because of a limited number of storage tanks, which often have to be shared by a multitude of products. Of course, each of these tanks also has its own capacity constraint (its maximum content). Furthermore, batches can not be stored concurrently, due to quality and traceability requirements. These constraints become even more relevant if the number of products is greater than the number of tanks, or if not all products can be stored in every tank.

The second storage limitation, time, is present due to the perishability of the intermediate food product. Unpackaged intermediate products are often more perishable than packaged products, which makes the storage time constraint in the intermediate stage of the production process more important than in other stages (raw material, final products). Within a certain time period, the product has to be packaged and transported to the customer, or else the product has to be disposed of as waste or low-quality by-product.

There are many practical situations where the two types of storage constraints are encountered. For example, in the production of dairy products, the customer often demands a certain best-before date. Thus, the possible storage time of perishable intermediates in the production process is very short. Another example is the production of flour, where intermediates have to be stored in a limited amount of 
silos. Due to different grains and different mixtures, the number of intermediates is very large, which can result in blocking effects caused by tank unavailability.

In the literature (discussed in section 2), intermediate storage is often considered as one single capacity constraint and the time constraint is hardly covered. This paper studies production systems with both types of constraints. We study the performance of a specific two-stage system under these constraints, and use several well-known (common-sense) heuristic sequencing approaches. For this study, we aim to explore the impact of the intermediate storage constraints. We believe a better understanding of the implications of these storage constraints is necessary as a starting point for the design of solution procedures for scheduling problems. We emphasize that in this paper, it is not the aim to develop a specific mathematical model and solve this to optimality. Instead, we consider this an exploratory study using a relatively simple stylized production system (but representing all (real-life) complexities in terms of interactions between production capacities and intermediate storage). We use simulation to investigate the behaviour under various capacity and time constraints on intermediate storage. We focus on several performance measures such as flow time, makespan, and waste. Underlying these measures, blocking and starvation effects play an important role. In the experiments, we also study the effect of uncertainty in processing times -as this is expected to influence blocking and starvation effects.

The paper is organised as follows. First, we discuss previous results on twostage production systems. Then we outline the production system we study in this paper and several heuristic scheduling approaches to be used in scheduling this production system. Subsequently, we present the results of simulation studies, which is the main contribution of this paper. Finally, the last sections will contain a discussion of the results and suggestions for further research. 


\section{Literature background}

There is a significant amount of studies that concern two-stage production systems. These systems already offer considerable complexity, as demonstrated by Gupta (1988). Johnson (1954) was one of the first to study such a system, with one machine in each stage. More recently, most papers address systems with one machine in the first stage and multiple machines in the second stage (e.g. Gupta and Tunc, 1991; Tsubone et al., 1996; Li, 1997). This type of problem is often found in process industries (Narasimhan and Panwalkar, 1984), and it resembles the typical divergent structure of production processes found in the food-processing industry (Akkerman and Van Donk, to appear).

A lot of studies, however, do not consider limited intermediate storage possibilities between the production stages. In the food-processing industries, we can distinguish capacity and time constraints on intermediate storage. Capacity constraints have been considered in several publications. In most cases, the limitation is included as an overall capacity constraint (e.g. Papadimitriou and Kanellakis, 1980; Nowicki, 1999), but several papers incorporate storage in the form of tanks (e.g. Belarbi and Hindi, 1992; Yi et al., 2000). From these papers, we learn that complex scheduling problems arise, which often can only be solved heuristically. We also see that tank availability is a specific concern in such situations and a main element in the modeling. Time constraints also received some attention in previous research, but relatively few studies consider this constraint (e.g. Yang and Chern, 1995; Su, 2003). Here we see that the time constraint dominates the development of heuristics and tighter constraints require more calculation efforts. To the best of our knowledge, the specific combination of capacity and time constraints has not been addressed in the literature. Furthermore, the complexity of the scheduling problems and the inherent difficulties in developing solution methods leads us to believe a good insight in this combination of constraints is important.

In our study, the first stage concerns a batch process. In the literature, the 
concept of batching is used in different ways. First, due to efficiency reasons, it can be convenient to process several jobs in a batch instead of processing them individually (see e.g. Potts and Kovalyov, 2000). For example, setup times can be involved when switching between product families. Then, the batching is the result of scheduling reasons and is called family scheduling (Webster and Baker, 1995). The main issue in family scheduling is the trade-off between minimal setup times and the order delivery time. Large batches delay the processing of orders from other product families (Potts and Kovalyov, 2000). Secondly, batching can also have technical reasons. In process industries, the processing stage often concerns non-discrete products, and processing technology often implies the need for batching. Then, a batch can be defined as a quantity that is planned to be produced in a given time period based on a formula or recipe that often is developed to produce a given number of end items (Cox and Blackstone, 2002). The batch sizes usually depend on the capacity of the batch processor. This is identified as batch processing (Webster and Baker, 1995).

In the above terminology, the kettle process in this paper is a batch processing machine. However, in our case, the sequencing of kettles is another relevant issue, because we assume setup times between product families. Therefore, scheduling the kettle process in this paper includes elements from batch processing and family scheduling. Furthermore, the intermediate storage time limitations we include also influence the coordination of the scheduling of the kettle process (see also Silver, 1989). In the field of chemical engineering, we also find various approaches to the scheduling of batch processing operations, mostly based on mixed integer linear programming (MILP) techniques (see e.g. Kondili et al., 1993; Pinto and Grossmann, 1998). Although these papers provide sophisticated production scheduling procedures, these approaches do not provide a thorough insight in the effects of constrained intermediate storage. 


\title{
3 Problem formulation
}

\subsection{Production system}

Figure 1 illustrates the production process studied in this paper. It is based on experiences in the food-processing industry, where such two-stage processing and packaging systems are very common.

[Figure 1 about here.]

\begin{abstract}
Although it is a relatively simple system, it contains all basic elements that determine the complexity of two-stage food production. In this way, we think that the results of the study provide general insights into the interactions and characteristics found in two-stage systems with intermediate storage in the food-processing industry.
\end{abstract}

The first stage consists of a kettle process, where $J$ different intermediate products are produced. The kettle content $B$ is fixed and it requires a processing time $p_{1, j}$ to produce one kettle of intermediate product $j$. This processing time is subject to uncertainty, due to variation in the quality of the raw material. In the foodprocessing industry, the raw material usually originates from the agricultural sector. These materials often have a variable quality by nature. During the processing time, the product stays in the kettle, and can only be transported to a storage tank after processing ends.

If multiple kettles of one product are produced in one batch, no setup time is required between these kettles. However, when changing to another product, a sequence-independent - setup period $S_{u p}$ is necessary. In this paper, a number of consecutively produced kettles of one product is referred to as a batch.

There are $K$ storage tanks, which can each hold $M$ finished kettles of an intermediate product. Two types of constraints are present for the intermediate storage: (i) a capacity constraint, concerning the number of tanks and their maximum content, and (ii) a storage time constraint. The capacity constraint is influenced by 
the fact that it is not allowed to store product from kettles in different batches in one storage tank, due to traceability issues. Due to this separation of batches, it is more likely that intermediate storage tanks are unavailable for a new batch.

The storage time constraint is related to the customer's requirements concerning the best-before date of a final product. To ensure a long best-before date, the intermediate product that is used for an end product has to be packaged within a maximum time $T_{\max }$. If several kettles of a product are stored in the tank, the finishing time of the first kettle determines the maximum storage time.

In the second stage of the production system, $J$ packaging lines are available to create various end products from each of the $J$ intermediate products. The unit processing time $p_{2, j}$ varies due to differences in packaging sizes. For small packaging sizes, it takes more time to package a certain amount of product, than for larger packaging sizes.

Finally, for reasons of simplicity, we make the following assumptions:

- The transport times between the batch processor and the storage tanks are negligible.

- Withdrawing product from tanks for packaging can only begin after batches are finished.

- Every storage tank can be used to store every intermediate product.

- Raw materials and packaging materials are always available.

- Storage of finished products is not relevant, as all products are immediately shipped to the customer.

- The production system operates only on weekdays, for eight hours per day.

\subsection{Product flow and scheduling}

Customer orders for the end products arrive during working days, and have to be delivered the next day. The number of orders $O_{j}$ for each product family $j$ varies 
from day to day. All orders also have their own packaging format requirements, which will be relevant in scheduling the packaging stage. At the start of each day, all orders become available to the planning department. At this time, schedules for the first and second stage can be created based on the orders and the current intermediate storage levels. However, this is not the only moment scheduling decisions are made. When the kettle process in the first stage has finished producing the intermediates that are needed to package the set of orders for the current day, it can be considered to make intermediate product for the next day. This is based on whether there is time left on the day and whether intermediate storage space is available.

In the first stage, a cyclic scheduling approach is adopted. According to Pinedo (2002), this is often the case in flow lines with limited intermediate storage. The approach is also attractive, because it will periodically supply different intermediate products to the second part of the production system, providing inputs for the packaging stage. The setup frequency for each intermediate product is denoted by the design parameter $S_{f}$, which is equal to the amount of cycles per day.

In each cycle, every product is produced once. The amount of kettles in a batch depends on the amount of products requested by the customer and the usable amount of product in the intermediate storage (where the usability is derived from the time constraint). We use $L_{j}$ to denote the amount of kettles of product $j$ that are needed on a certain day. This amount can be calculated by ${ }^{1} L_{j}=\left\lceil\left(O_{j}-U_{j}\right) / B\right\rceil$, where $B$ is the kettle content, $U_{j}$ is the usable amount of product $j$ which is in the intermediate storage at the start of the day, and $O_{j}$ the amount of products from family $j$ to be produced that day - collected on the day before. Finally, these $L_{j}$ kettles are divided between the production cycles. That means that the $p^{\text {th }}$ cycle for product $j$ (called $\left.C L_{j p}\right)$ has $\left\lceil\left(L_{j}-\sum_{i=1}^{p-1} C L_{j i}\right) /\left(S_{f}-(p-1)\right)\right\rceil$ kettles.

For the additional production at the end of the day, this cyclic scheduling ap-

\footnotetext{
${ }^{1}$ The notation $\lceil x\rceil$ is defined as the smallest integer greater then or equal to the value of $x$, or mathematically speaking: $\lceil x\rceil=\min \{y \mid y \in \mathbf{N}, y \geq x\}$.
} 
proach will be continued. However, the batch size (in kettles) cannot be based on customer demand, because this information is not available until the next day. Therefore, it is based on a forecast of the requested orders for the next day. On Fridays, no additional production is scheduled, because due to the storage time constraint, the product would be unusable on the next Monday. As compensation, the kettle process is started earlier on Mondays. For the remaining weekdays, production at the end of the previous day can be used and starting earlier is not necessary. In this way, the production is mostly done during the regular working hours, which can be economically attractive.

In the second stage, the intermediate product is packaged to satisfy the customer orders. Because of the varying packaging times, the packaging sequence influences the speed at which product is extracted from the tanks. In this paper, we use three different ways to sequence the production in this stage:

- FCFS rule (First Come, First Serve), where the orders are processed in the order they arrive. The main idea behind the inclusion of this rule is its usefulness as a benchmark. In many cases it is also attractive because it results in a low variance of flow time (see e.g. Rajendran and Holthaus, 1999).

- SPT rule (Shortest Processing Time), which arranges the products according to an ascending order of unit processing times. This rule is traditionally seen as the best rule in terms of flow time (see e.g. Holthaus and Rajendran, 2002), although some authors discuss its effectiveness in situations with bottlenecks (see Bassett and Todd, 1994).

- LPT rule (Longest Processing Time), which arranges the products according to a descending order of unit processing times. According to Tsubone et al. (1996), this rule yields good results in terms of the maximum work-inprocess level, which makes it especially interesting to consider in a situation where storage is constrained (in capacity and time). 
The storage constraints are an important characteristic of this production system. Both the production cycle in the first stage and the packaging sequence in the second stage interact with these constraints. This interaction results in blocking and starvation effects. Blocking occurs when the kettle process finishes, but there is no intermediate storage tank available. This means the product stays in the kettle until a tank becomes available and therefore temporarily blocks further production. The blocking effects are strengthened by the traceability requirements mentioned before. Even a small amount of the same product as in the kettle could block a storage tank. Starvation occurs when there are customer orders to package, but the required intermediate product is unavailable. Then, the packaging line is idle until the intermediate product becomes available.

If there is much blocking and starvation, it can be the case that it is not possible to satisfy all customer orders within the time available.

\subsection{Illustrative example}

To clarify the characteristics of the production system, figure 2 shows an example of a production schedule for a system with three packaging lines, three storage tanks with a content of three kettles $(J=3, K=3, M=3)$.

[Figure 2 about here.]

In this example, we present a situation where two production cycles are processed in the first stage $\left(S_{f}=2\right)$ and the intermediate storage tanks are empty at the start of the day. The storage time constraint is assumed to be nonrestrictive. The impact of the capacity constraint is visible through blocking and starvation effects. For each of the product families, five kettles of intermediate product are needed to satisfy demand $\left(L_{j}=5, \forall j\right)$.

Starvation is seen at the start of the day, and during the day after the packaging of the first batches. Blocking effects can be seen in the first stage. After the production of the first kettle in the second batch of product 3 , there is no storage 
tank available. Only after a tank becomes available, the content of the kettle can be moved to this storage tank.

This example also provides insight into the effect of production in advance. At the end of the day, there is sufficient time to start with a new production cycle. However, there is a reasonable chance that blocking effects will occur, due to the situation that no storage tank is available. In the example, one additional batch of product 1 is produced to create a starting inventory for the next day. If this is possible (considering capacity and time constraints), there is a significant time advantage on the next day. This advantage is that one of the packaging lines does not have to wait until the batch processor finishes the first batch, which reduces the amount of starvation.

\subsection{Performance criteria}

For the formulation of the performance criteria, we introduce several additional variables. For the first stage, let $S_{1, j l}$ and $C_{1, j l}$ be the starting time and completion time for the $l^{\text {th }}$ kettle process of intermediate product $j$ (from a total of $L_{j}$ kettles). For the second stage, we define $S_{2, j o}$ and $C_{2, j o}$ to be the starting time and completion time for the packaging of the $o^{\text {th }}$ order an end product from family $j$. As defined earlier, we use $O_{j}$ to denote the number of orders for product $j$ (for the current day).

First, we will use daily flow time, makespan, and the amount of unfinished orders to evaluate the production systems ability to finish the requested orders. With the flow time, we have a indication of the time the orders spend in the production process, which (for practical considerations) translates into lead times for individual orders. The makespan gives us an idea on the total time needed to finish the daily production. We denote these criteria by $F T, M S$, and $U F$ and calculate them as follows:

$$
F T=\sum_{j=1}^{J} \sum_{o=1}^{O_{j}} C_{2, j o},
$$




$$
U F=\sum_{j=1}^{J} \sum_{o=1}^{O_{j}} I_{j o}^{u}
$$

where $I_{j o}^{u}$ is an indicator function, defined as follows:

$$
I_{j o}^{u}= \begin{cases}1, & \text { if the } o^{\text {th }} \text { order for a product from family } j \text { is unfinished } \\ & \text { at the end of the day } \\ 0, & \text { otherwise. }\end{cases}
$$

Due to the limited storage capacity, we also measure the amount of blocking in the first stage and the amount of starvation in the second stage to evaluate the systems performance. These criteria will provide insight on the effects of the limited number of storage tanks between the two stages. In this formulation, we let $T_{1, j l}$ denote the moment in time that the $l^{\text {th }}$ kettle for family $j$ is transported to an intermediate storage tank. The amount of blocking is then calculated in the following way:

$$
B L=\sum_{j=1}^{J} \sum_{l=1}^{L_{j}}\left(T_{1, j l}-C_{1, j l}\right),
$$

and the amount of starvation as follows:

$$
S T=\sum_{j=1}^{J}\left(\sum_{o=2}^{O_{j}}\left(S_{2, j o}-C_{2, j(o-1)}\right)+S_{2, j 1}\right) .
$$

The other additional element in this paper is the limited waiting time of products in the intermediate storage. Therefore, it can happen that a batch of intermediate product becomes obsolete and unusable for further production. The last performance criterion we include is therefore the amount of waste. Here, $S L_{k}$ and $F D_{k}$ denote the storage level of product in tank $k$ and the fill date of tank $k$. We measure the time constraint on intermediate storage in days, so the waste on a certain day $d$ can be calculated as follows:

$$
W A=\sum_{k=1}^{K} I_{k}^{w} S L_{k}
$$


where $I_{k}^{w}$ is defined as follows:

$$
I_{k}^{w}= \begin{cases}1, & \text { if } d-F D_{k}>T_{\max } \\ 0, & \text { otherwise. }\end{cases}
$$

\section{Numerical experiments}

Several experiments have been performed to analyse the performance of various configurations of the production system. The aim of these experiments is to study the influence of several intermediate storage constraints, and to study the applicability of different sequencing rules in the packaging stage.

As was mentioned in Section 3, there are several elements subject to uncertainty. In the simulation study, these are inserted as follows. The batch processing time $p_{1, j}$ is generated from a truncated normal distribution with average $\bar{a}$ and coefficient of variance $c v_{a}$.

The customer orders for products from family $j$ arrive during the day following a Poisson distribution with an average of $\lambda$ units per day. For the second stage, the unit processing time $p_{2, j}$ is dependent on the packaging requirements of the customer and is randomly set at $b_{\min }$ or $b_{\max }$, where $b_{\min }=\bar{b}-b_{\mathrm{dev}}$ and $b_{\max }=\bar{b}+b_{\mathrm{dev}}$. With this implementation, we are also able to study the effect of smaller and larger differences in packaging times by varying $b_{\mathrm{dev}}$.

We expect that the uncertainty in the processing times has a significant impact on the performance of the production system. Variation in the moment that products are transported to the intermediate storage tanks and the moments that they are extracted from these tanks influences blocking and starvation effects and will therefore affect the systems performance.

Next to the effect of uncertainty, we will also study various configurations of the intermediate storage system. We will study capacity-constrained intermediate storage by looking at different numbers of storage tanks, as we are interested to see to what extent there is an effect on the performance criteria through increased 
or decreased blocking and starvation.

Finally, we also look at time constraints for the intermediate storage. We expect tight storage time constraints to have a big influence on the performance of the sequencing heuristics and also the choice for a specific setup frequency.

The parameter settings used in the initial model are listed in table 1.

[Table 1 about here.]

The simulation results in the following section have all been derived from 100 simulation runs of 10 weeks. It should be noted that, due to the storage time constraint, the products in the intermediate storage cannot be stored over the weekend. This results in independence between weeks. Therefore, the run length in weeks is arbitrary, as long as it is a number of full weeks.

\section{Simulation results}

\subsection{Effects of uncertainty}

To evaluate the effect of the sequencing rules used in the second stage, we will first look at various differences between the processing times by varying $b_{\mathrm{dev}}$. Greater variation in these processing times should increase the effects of using a certain sequencing rule. All other parameters have the values listed in table 1.

In figure 3 , the flow time and makespan are shown for various values of $b_{\mathrm{dev}}$ and various sequencing rules. The value of $b_{\mathrm{dev}}$ represents the variation in packaging times.

[Figure 3 about here.]

As can be seen in these figures, the amount of variation in the packaging time in the second stage has significant effects on the flow time and the makespan. First, using certain sequencing rules becomes more important. The result from figure 3(a) can be explained by the definition of flow time (summation of finishing 
times). If all orders with small processing times are processed first, it is obvious that a sum of completion times is smaller then if orders with long processing times are processed first. However, despite the disadvantage in flow time, the makespan is lower for the LPT sequencing rule (as shown in figure 3(b)). This is due to an increase in the amount of starvation time; because when the second stage finishes packaging relatively soon (SPT rule), it has to wait for new intermediates to continue. The amount of blocking time is relatively constant, due to the fact that this also occurs before or during the additional production. However, this part of the blocking time does not affect the packaging of customer orders.

Next, the effect of uncertainty in the batch processing time is studied. With more uncertainty, we expect that the chances of blocking and starvation increase, which in turn could effect our main performance criteria, like flow time and makespan. Therefore, in figure 4, the flow time and makespan is shown for various values of $s d_{a}$, which is the standard deviation of $\bar{a}$.

[Figure 4 about here.]

More variation in the batch processing time increases the flowtime and the makespan of the production system. This can be explained as follows. If the variation in the batch processing time increases, we both get batches that take a longer time and batches that finish faster. In the first case, makespan and flow time are negatively influenced. In the second case, this does not necessarily have to be true. It is possible that no intermediate storage capacity is available and blocking effects occur. This increase in blocking is partly cancelled by a decrease in blocking effects due to the longer processing times. However, these longer processing times also result in an increase in starvation time. Overall, the result is an increase in flow time and makespan, as was shown in figure 4. 


\subsection{Effect of the number of tanks}

In figure 5, the flow time and makespan performance criteria are shown for different numbers of storage tanks.

[Figure 5 about here.]

With the increase of the amount of intermediate storage tanks, there is (initially) an improvement in these performance criteria. This is mostly due to a decrease in the blocking and starving time encountered. For more than six storage tanks (two for each family), not much more improvement is seen.

[Figure 6 about here.]

However, as we can see in figure 6(a), the addition of only one storage tank already results in reducing the amount of unfinished orders to almost zero. With more than six storage tanks, there is even a slight increase in the amount of unfinished orders. This is likely to be caused by an increase in waste, as is shown in figure 6(b).

\subsection{Effect of the storage time constraint}

The storage time constraint has a significant effect on the production systems' performance. Until now, we used a maximum storage time of one day. Here we will consider the performance for different values of $T_{\max }$, which we will now state in minutes. The value of $T_{\max }$ also has an important effect on the scheduling; if the time constraint is getting tighter, it is not possible anymore to produce intermediate product for the next day.

As the storage time constraint becomes tighter, it seems logical to reduce batch sizes (increase setup frequency). In this way, smaller amounts of intermediate product are delivered to the storage tanks and can subsequently be packaged faster. Therefore, we look at different setup frequencies. Figure 7 shows the flow 
time and makespan for various values of $T_{\max }$ for three different setup frequencies. The initial setup frequency $S_{f}$ in our study is 2 . In the simulation, we also used $S_{f}=3$ and $S_{f}=4$.

[Figure 7 about here.]

In the two figures, there is a jump at around 1000 minutes. This change is due to the possibility of storing product overnight when the time constraint is above 1000 minutes. As we can see, being able to 'work in advance' reduces the flow time and makespan significantly.

Several interesting results can be seen in the figures. First, the possibility to store products overnight makes the choice of setup frequency very relevant. For tighter storage constraints, it is useful to increase the setup frequency from 2 to 3. A higher setup frequency results in a lot of setup time and increases makespan.

Secondly, the difference in flow time and makespan with and without the possibility to 'work in advance' is quite big. For the makespan (figure 7(b)), note that it is only possible to produce the given orders in a shift of eight hours (480 minutes) if the storage constraint allows to work in advance for the next day. When this is not the case, extensive starving effects at the start of the day negatively influence the performance criteria (as was also illustrated in the example in figure 2).

[Figure 8 about here.]

Finally, the amount of waste (figure 8) is significantly higher if the storage constraint does not allow storing overnight. This can be explained by the fact that, on every day, all remaining intermediate storage has to be disposed of as waste. Around $T_{\max }=1000$, it can be seen that the setup frequency has quite an effect on the amount of waste.

For small values of $T_{\max }$, the sequencing rule used is again important. In figure 9(a), the number of unfinished orders in shown for different values of $T_{\max }$. It can be seen that with the SPT rule, almost all order are fulfilled for $T_{\max } \geq 160$ minutes. 
For the LPT rule, this is true for $T_{\max } \geq 200$ minutes. The amount of unfinished orders are also resembled in the amount of waste, as shown in figure 9(b).

[Figure 9 about here.]

An explanation for this behaviour can be found in the fact that with the LPT rule the packaging of the first batch can take more time than the production of the following batch. This can in turn cause blocking effects which means that a product already starts its decay before it is even transferred to the intermediate storage.

\section{Conclusions and further research}

In this paper, we addressed intermediate storage tanks with capacity and storage time constraints in a two-stage production system with a batch processor in the first stage and several packaging lines in the second stage. The combination of capacity and time constraints has not been studied before. Our contribution in this paper is seeing how several common-sense scheduling and sequencing rules perform in the presence of these constraints, and analysing how various capacity and time constraints influence the systems performance.

First, the type of sequencing rule in the second stage has significant effects on performance criteria like makespan and flow time. This effect is stronger as the variation in packaging times is higher. Although flow time is minimised by using the SPT rule, the makespan is minimised by the LPT rule. The latter is caused by less starvation in the packaging stage, and can also result in an overall increase in production volume per day. This is an interesting result, because of the intuitiveness of using the SPT rule to empty the storage tanks as soon as possible.

Secondly, we conclude that to manufacture an acceptable number of orders, the number of tanks should be at least equal to the number of packaging lines.

When adding one additional tank above that number, almost all orders can be 
finished in time, but there still are some blocking and starvation effects, which influence the time needed to finish the set of orders. From our analysis, it follows that more additional storage tanks reduce flow time, makespan, blocking time, and starvation time. This effect decreases significantly with every additional storage tank. However, increasing the number of tanks does result in more waste, due to violation of the time constraint. If the number of tanks is more than twice the amount of packaging lines, the increase in waste results in more unfinished orders. This is mainly caused by the need to produce additional batches in the processing stage to replenish the waste. Interestingly, this means that adding tank capacity to the production system could negatively influence some performance measures.

Finally, the storage time constraint has been varied and it clearly shows that it is beneficial to use a different setup frequency if it is not possible to store the intermediate product until the next day. For different storage time constraints, using different sequencing heuristics only has influence for tighter constraints. The SPT rule can cope with tighter storage time constraints than the other rules because of less blocking. There is more starvation, but this does not affect product perishability and waste. As seen earlier, starvation is more important when considering performance criteria like makespan.

The managerial implications of these results could be summarised as follows. First, the intuitive idea of emptying the intermediate storage tanks as soon as possible has a significant drawback in terms of makespan (through increased starvation time). Secondly, the results show that one additional storage tank already has a significant impact on the system performance. Additional tanks can be used to further decrease flow time or makespan, but these investments should carefully be considered. Also, adding tank capacity can lead to more waste and unfinished orders, which is something that needs to be carefully monitored. Third, for tight storage time constraints, emptying the storage tanks as soon as possible does result in the lowest amount of unfinished orders. It turns out that, under tight storage 
constraints, it is important to realize that both blocking and starvation negatively effect the performance of the production system in terms of flow time and makespan, but only blocking is relevant in causing waste. Finally, an analysis as the one described in this paper is a very useful tool for evaluating the effects of design or expansion decisions.

We realise that the first and the third implication represent a trade-off. If one has to implement a sequencing policy in practice, both implications should be considered and choices have to be made depending on the situation. The development of tools to support managers in making such decisions is an interesting direction for further research.

The results in this paper are based on specific production characteristics. This also raises interesting directions for further research. For instance, we assumed a symmetrical demand pattern for the different product families. Studying the impact of a non-symmetrical demand pattern would be an interesting next step. Also, an interesting suggestion would be to include a distinction between storage tanks that are dedicated to a single product and storage tanks that can be used for multiple products. This would also result in more capacity constraints for the intermediate storage, which could require special treatment in the scheduling and sequencing process. Finally, the analysis could be extended to include other characteristics of the food-processing industry, such as random yields or sequencedependent setup times.

\section{References}

AKKERMAN, R. and VAN DONK, D.P., to appear, Analysing scheduling in the food-processing industry: Structure and tasks, Cognition, Technology E Work, (accepted for publication).

BASSETT, G. and TODD, R., 1994, The SPT priority sequence rule, International 
Journal of Operations \& Production Management, 14(12), 70-78.

BELARBI, K. and HINDI, K.S., 1992, Detailed scheduling for a class of two-stage intermittent manufacturing systems, Production Planning $\mathcal{E}$ Control, 7(1), 36-47.

COX, J.F. and BLACKSTONE, J.H. (eds.), 2002, APICS Dictionary (Alexandria, VA: American Production and Inventory Control Society), 10th edition.

GUPTA, J.N.D., 1988, Two-stage, hybrid flowshop scheduling problem, Journal of the Operational Research Society, 39(4), 359-364.

GUPTA, J.N.D. and TUNC, E.A., 1991, Schedules for a two-stage hybrid flowshop with parallel machines at the second stage, International Journal of Production Research, 29(7), 1489-1502.

Holthaus, O. and Rajendran, C., 2002, A study on the performance of scheduling rules in buffer-constrained dynamic flowshops, International Journal of Production Research, 40(13), 3041-3052.

JOHNSON, S.M., 1954, Optimal two- and three-stage production schedules with setup times included, Naval Research Logistics Quarterly, 1, 61-68.

Kondili, E., PAntelides, C.C., and SARgent, R.W., 1993, A general algorithm for short-term scheduling of batch operations - I. MILP formulation, Computers E Chemical Engineering, 17(2), 211-227.

LI, S., 1997, A hybrid two-stage flowshop with part family, batch production, major and minor set-ups, European Journal of Operational Research, 102(1), 142-156.

NARASIMHAN, S.L. and PANWALKAR, S.S., 1984, Scheduling in a two-stage manufacturing process, International Journal of Production Research, 22(4), 555-564.

NOWICKI, E., 1999, The permutation flow shop with buffers: A tabu search approach, European Journal of Operational Research, 116(1), 205-219. 
Papadimitriou, C.H. and Kanellakis, P.C., 1980, Flowshop scheduling with limited temporary storage, Journal of the ACM, 27(3), 533-549.

PINEdo, M., 2002, Scheduling: Theory, Algorithms, and Systems (Upper Saddle River, New Jersey: Prentice Hall), second edition.

PinTO, J.M. and GrossmanN, I.E., 1998, Assignment and sequencing models for the scheduling of process systems, Annals of Operations Research, 81, 433-466.

PotTs, C.N. and Kovalyov, M.Y., 2000, Scheduling with batching: a review, European Journal of Operational Research, 120(2), 228-249.

RAJENDRAN, C. and Holthaus, O., 1999, A comparative study of dispatching rules in dynamic flowshops and jobshops, European Journal of Operational Research, 116(1), 156-170.

SILVER, E.A., 1989, Shelf life considerations in a family production context, International Journal of Production Research, 27(12), 2021-2026.

SU, L.-H., 2003, A hybrid two-stage flowshop with limited waiting time constraints, Computers $\mathcal{E}$ Industrial Engineering, 44(3), 409-424.

Tsubone, H., OhbA, M., and Uetake, T., 1996, The impact of lot sizing and sequencing on manufacturing performance in a two-stage hybrid flow shop, International Journal of Production Research, 34(11), 3037-3053.

VAN DONK, D.P., 2001, Make to stock or make to order: The decoupling point in the food processing industries, International Journal of Production Economics, 69(3), 297-306.

WEBSTER, S.T. and BAKER, K.R., 1995, Scheduling groups of jobs on a single machine, Operations Research, 43(4), 692-703. 
YANG, D.-L. and CHERN, M.-S., 1995, A two-machine flowshop sequencing problem with limited waiting time constraints, Computers $\mathcal{E}$ Industrial Engineering, 28(1), 63-70.

YI, G., SUH, K.-H., LEE, B., and LEE, E.S., 2000, Optimal operation of quality controlled product storage, Computers $\mathcal{E}$ Chemical Engineering, 24(2-7), 475-480. 


\section{List of Figures}

1 General form of the two-stage production process with a batch processor in the first stage and $J$ parallel packaging lines in the second stage. . . . . . . . . . . . . . . . . 25

2 Schedule for the example case. . . . . . . . . . . . . 26

3 Average flow time and average makespan against $b_{\mathrm{dev}}$ for various heuristics. . . . . . . . . . . . . . . . 27

4 Average flow time and average makespan against $s d_{a}$ for various heuristics. . . . . . . . . . . . . . . . 28

5 Average flow time and average makespan against $K$ for various heuristics. . . . . . . . . . . . . . . . . . . . . . 29

6 Average amount of unfinished orders and waste against $K$ for various heuristics. . . . . . . . . . . . . . . . 30

7 Average amount of unfinished orders and waste against $T_{\max }$ for various heuristics. . . . . . . . . . . . . . . . 31

8 Amount of waste against $T_{\max }$ for various setup frequencies. . . . . 32

9 Average amount of unfinished orders and waste against $T_{\max }$ for various heuristics. . . . . . . . . . . . . . . 33 


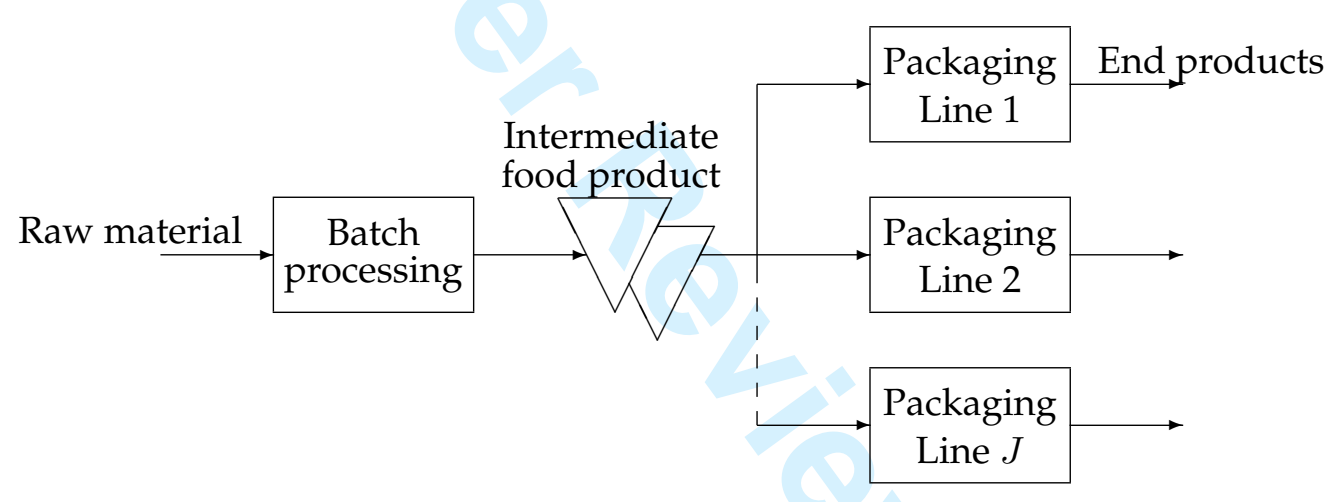

Figure 1. General form of the two-stage production process with a batch processor in the first stage and $J$ parallel packaging lines in the second stage. 


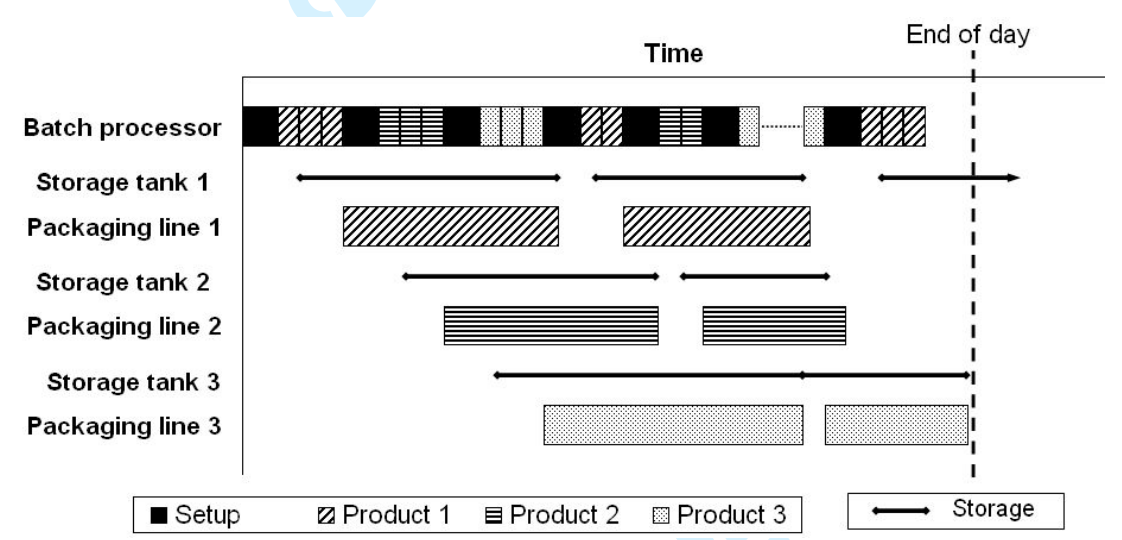

Figure 2. Schedule for the example case. 


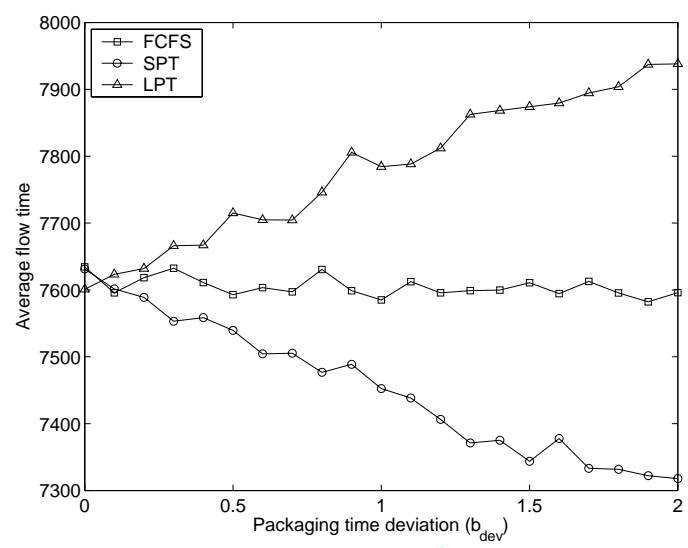

(a) Average flow time

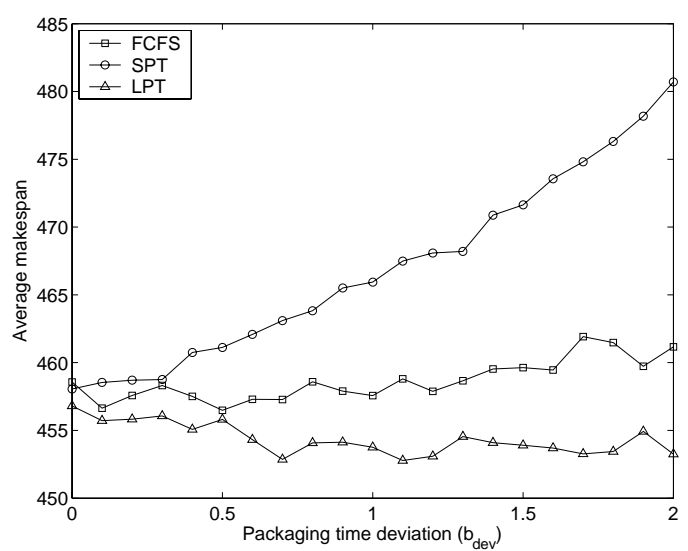

(b) Average makespan

Figure 3. Average flow time and average makespan against $b_{\mathrm{dev}}$ for various heuristics. 


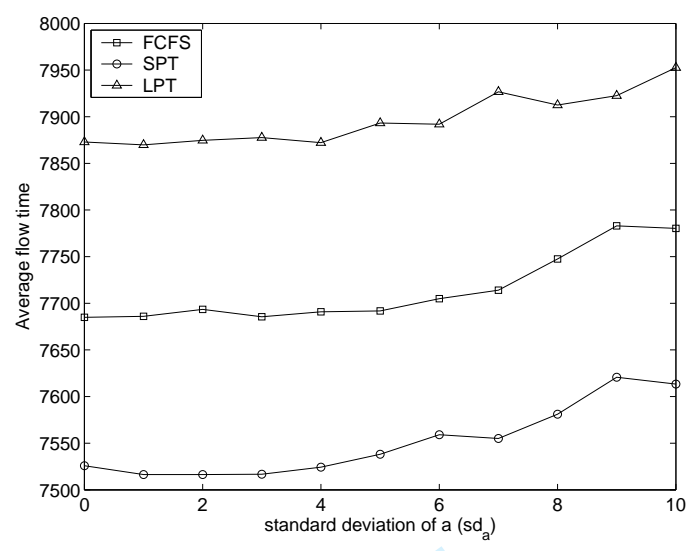

(a) Average flow time

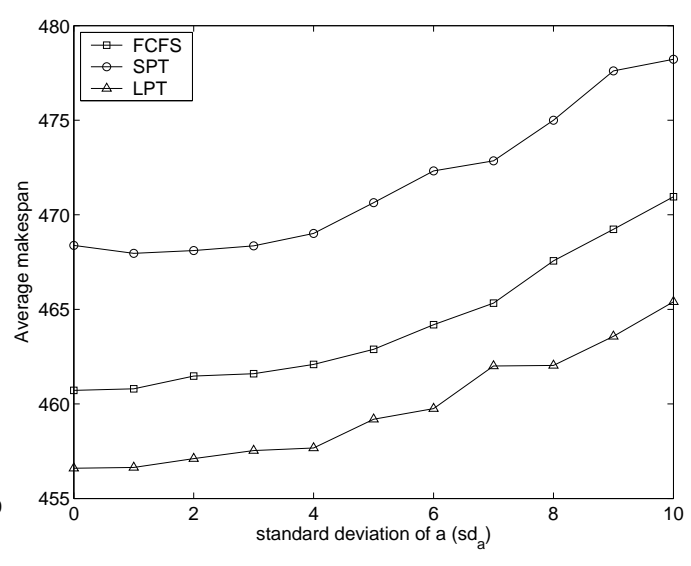

(b) Average makespan

Figure 4. Average flow time and average makespan against $s d_{a}$ for various heuristics. 


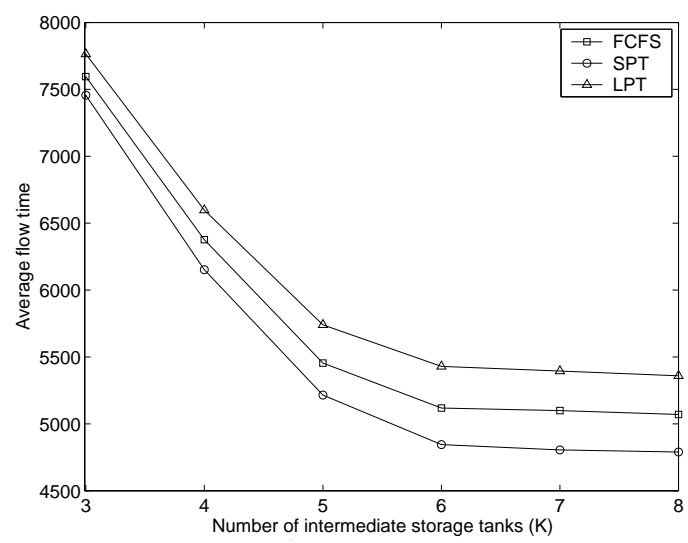

(a) Average flow time

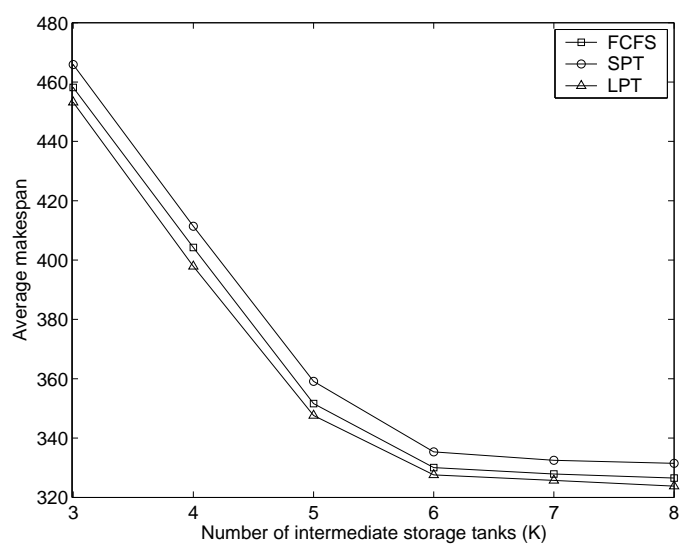

(b) Average makespan

Figure 5. Average flow time and average makespan against $K$ for various heuristics. 


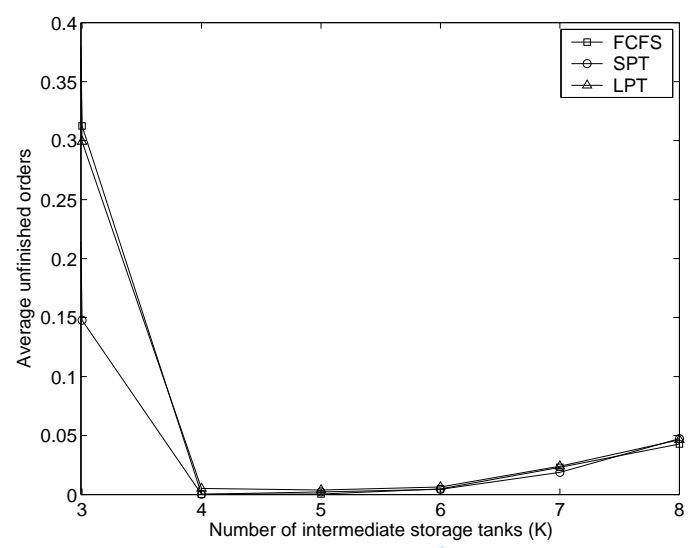

(a) Average number of unfinished orders

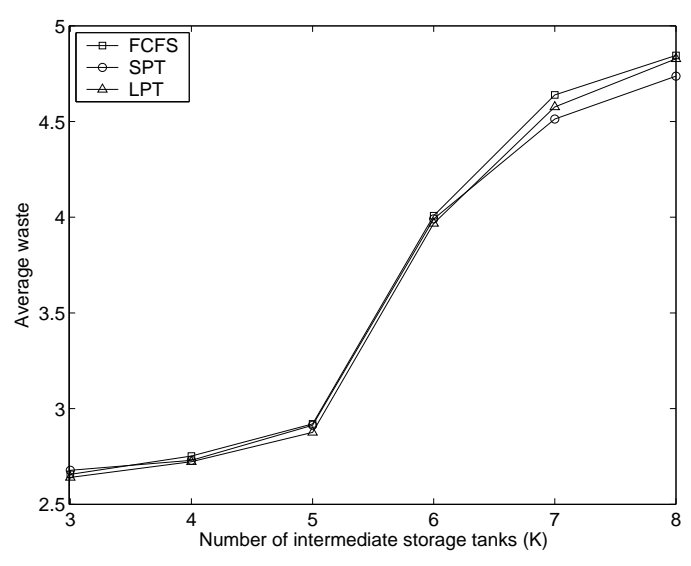

(b) Average amount of waste

Figure 6. Average amount of unfinished orders and waste against $K$ for various heuristics. 


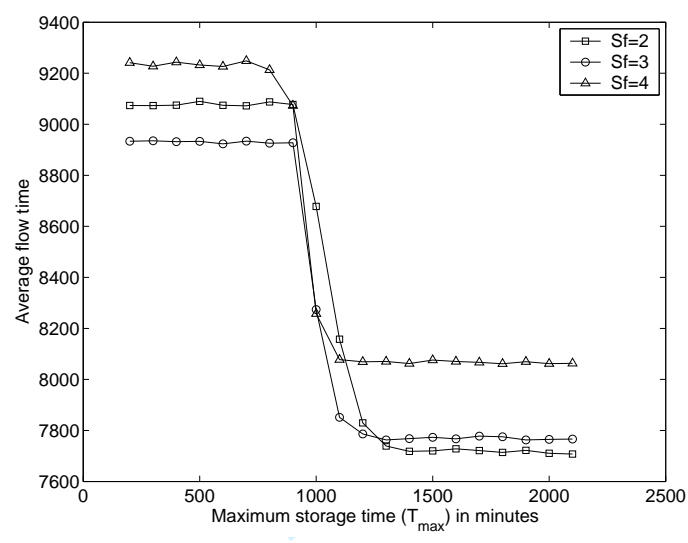

(a) Average flowtime

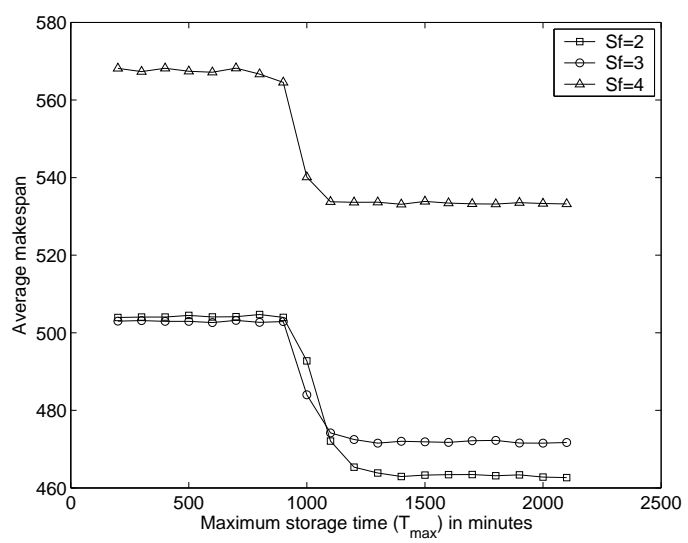

(b) Average makespan

Figure 7. Average amount of unfinished orders and waste against $T_{\max }$ for various heuristics. 


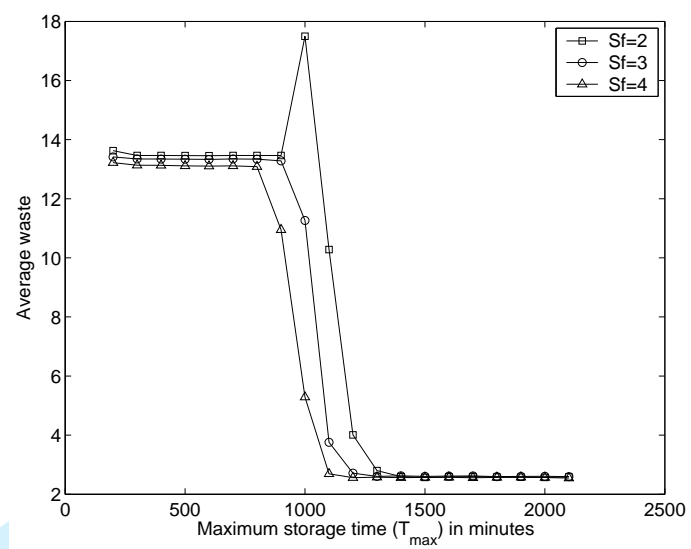

Figure 8. Amount of waste against $T_{\max }$ for various setup frequencies. 


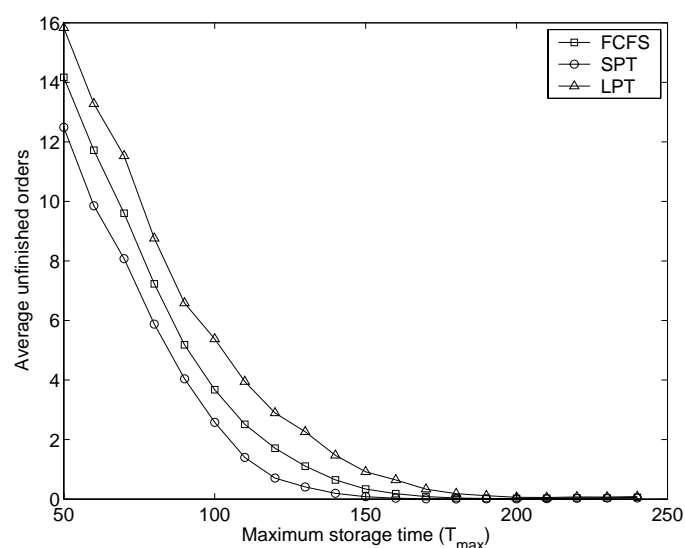

(a) Average number of unfinished orders

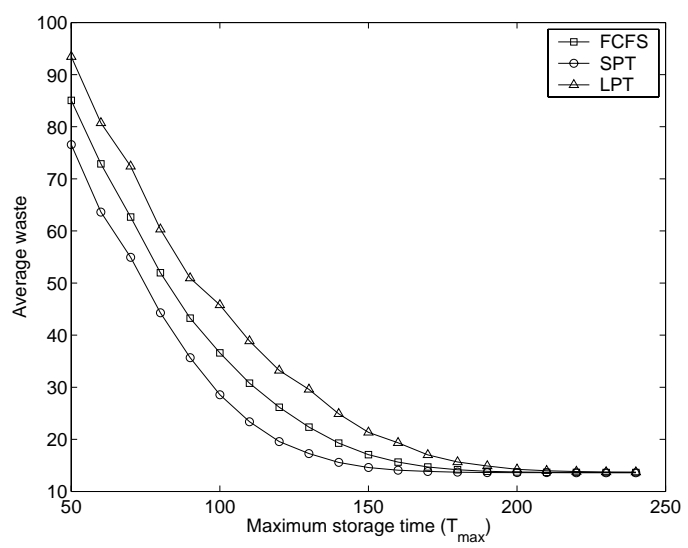

(b) Average amount of waste

Figure 9. Average amount of unfinished orders and waste against $T_{\max }$ for various heuristics. 


\section{List of Tables}

1 Initial values of parameters used in the model. . . . . . . . . . . 35 
Table 1. Initial values of parameters used in the model.

\begin{tabular}{clclcl}
\hline Parameter & Value & Parameter & Value & Parameter & Value \\
\hline$\lambda$ & 50 units /day & $K$ & 3 tanks & $\bar{a}$ & 15 minutes \\
$J$ & 3 families & $M$ & 5 kettles & $c v_{a}$ & 0.2 \\
$S_{u p}$ & 25 minutes & $B$ & 10 units & $\bar{b}$ & 5 minutes \\
$S_{f}$ & 2 cycles & $T_{\max }$ & 1 day & $b_{\mathrm{dev}}$ & 1 \\
\hline
\end{tabular}

